\title{
Introduction to DSmT for Information Fusion
}

\author{
Jean Dezert \\ ONERA/DTIM/IED
Av. de la Division Leclerc
Ch20 Châtillon, France \\ ONERA/DTIM/IED
29 Av. de la Division Leclerc
92320 Châtillon, France \\ ONERA/DTIM/IED
Av. de la Division Leclerc
92320 Châtillon, France
}

jean.dezert@onera.fr

jean.dezert@onera.fr
Florentin Smarandache

Department of Mathematics

University of New Mexico

Gallup, NM 8730, U.S.A.

smarand@unm.edu

Panel discussion on DSmT - 7th Int. Conf. on Information Fusion Fusion 2004 - Stockholm - Sweden 


\section{Outline}

- Introduction

- Foundations (Dedekind's lattice)

- Free, hybrid DSm models and Shafer's model

- Generalized belief functions

- DSmT rules of combination

- Dempster's rule and its existing alternatives

- Static and dynamic fusion

- Examples

Extension to the fusion of imprecise beliefs

- Summary of DSm fusion principle

- Practical limitations of DSmT

- Applications

- Conclusions 


\section{DSmT in few lines}

DSmT (Dezert-Smarandache Theory) is a new alternative to Dempster-Shafer Theory (DST) which :

I - proposes a new mathematical framework for information fusion

2 - incorporates any kinds of model (free, hybrid DSm models and/or Shafer's model) for taking into account any integrity constraints of the fusion problem

3 - combines uncertain, high conflicting and imprecise sources of evidence with a new rule of combination and overcomes limitations of the Dempster's rule

4 - is adapted to static or dynamic fusion applications represented in terms of belief functions based on the same general formalism 


\section{DSmT Foundations}

\section{Frame of the problem $\Theta=\left\{\theta_{1}, \theta_{2}, \ldots, \theta_{n}\right\}$. Finite set of exhaustive elements (discrete/continuous/fuzzy/relative concepts)}

Hyper-power set (Dedekind's lattice) $\quad\left|D^{\Theta}\right|>\left|2^{\Theta}\right|$

1. $\emptyset, \theta_{1}, \ldots, \theta_{n} \in D^{\Theta}$

2. $\forall A \in D^{\Theta}, B \in D^{\Theta},(A \cup B) \in D^{\Theta},(A \cap B) \in D^{\Theta}$

3. No other elements belong to $D^{\Theta}$, except those, obtained by using rules 1 or 2 .

Note : hyper-power set reduces to power set for the Shafer's model

Cardinalities d(n) of hyper-power sets follow Dedekind's sequence and elements can be generated by isotone Boolean functions. Hyper-power set is closed under union and intersection operators but is not a Boolean algebra.

Example for $\mathrm{n}=3 \quad \mathrm{~d}(\mathrm{n}=3)=19$

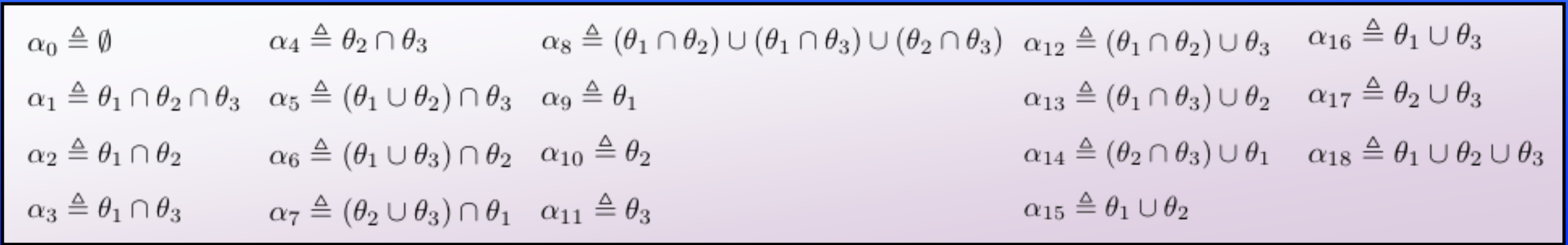




\section{DSm Models}

The granularity of the model of the frame characterizes the intrinsic nature (discrete/continuous, precise/vague,absolute/relative, etc) of the concepts involved in the fusion process.

\section{Free DSm model}

Elements of the frame are vague and potentially overlapping. Free = no constraint on elements. Useful to manipulate continuous concepts having relative interpretation (where ultimate refinement is inaccessible)

\section{Hybrid DSm model}

Some elements of the frame can be exclusive and/or non existing specially for dynamic fusion applications. Hybrid = introduction of integrity constraints into the free DSm model.

Parts have vague boundaries
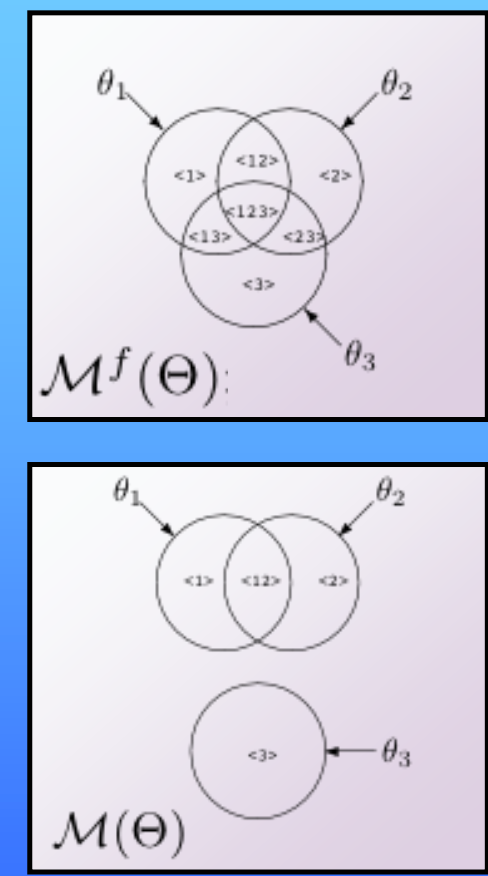

\section{Special hybrid model: Shafer's model}

All exhaustive elements of the frame are known to be truly exclusive (i.e. a refinement is accessible)

Constraints are represented by the characteristic nonemptiness function $\Phi(A)$ for all $A$ in hyper-power set.

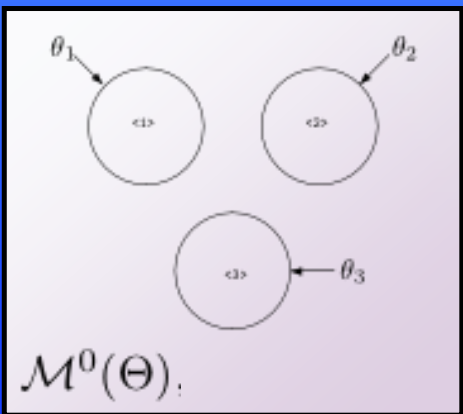

Parts have precise boundaries 


\section{Generalized belief functions}

Generalized basic belief assignment

$$
m(.): D^{\Theta} \rightarrow[0,1] \quad m(\emptyset)=0 \quad \text { and } \quad \sum_{A \in D^{\Theta}} m(A)=1
$$

Generalized belief/credibility function

$$
\operatorname{Bel}(A)=\sum_{\substack{B \subseteq A \\ B \in D^{\ominus}}} m(B)
$$

Generalized plausibility function

Generalized Pignistic Transformation (GPT)

$$
\operatorname{Pl}(A)=\sum_{\substack{B \cap A \neq \emptyset \\ B \in D^{\Theta}}} m(B)
$$

GPT allows to build a subjective probability measure P\{.\} over hyper-power set to help the decision making under uncertainty (but other issues are possible).

$$
\forall A \in D^{\Theta} \quad P\{A\}=\sum_{X \in D^{\Theta}} \frac{\mathcal{C}_{\mathcal{M}}(X \cap A)}{\mathcal{C}_{\mathcal{M}}(X)} m(X)
$$$$
\operatorname{Bel}(A) \leq P\{A\} \leq \operatorname{Pl}(A)
$$ 


\section{General DSm rule of combination}

For any DSm model, the fusion of $k$ independent equally* reliable sources of evidences is done by

$$
m_{\mathcal{M}(\Theta)}(A) \triangleq \phi(A)\left[S_{1}(A)+S_{2}(A)+S_{3}(A)\right]
$$

$$
\begin{aligned}
& S_{1}(A) \triangleq \sum_{\substack{X_{1}, \ldots, X_{k} \in D^{\Theta} \\
\left(X_{1} \cap \ldots \cap X_{k}\right)=A}} \prod_{i=1}^{k} m_{i}\left(X_{i}\right) \\
& S_{2}(A) \triangleq \sum_{\substack{X_{1}, X_{2}, \ldots, X_{k} \in \emptyset \\
[\mathcal{U}=A] \vee\left[(\mathcal{U} \in \emptyset) \wedge\left(A=I_{t}\right)\right]}} \prod_{i=1}^{k} m_{i}\left(X_{i}\right) \\
& S_{3}(A) \triangleq \sum_{X_{1}, X_{2}, \ldots, X_{k} \in D^{\Theta}} \prod_{i=1}^{k} m_{i}\left(X_{i}\right) \\
& \left(X_{1} \cup X_{2} \cup \ldots \cup X_{k}\right)=A \\
& \left(X_{1} \cap X_{2} \cap \ldots \cap X_{k}\right) \in \emptyset
\end{aligned}
$$

General DSm rule does not require normalization and is not equivalent to Dempster's rule.

Special case: Classic DSm rule for free DSm model This corresponds to conjunctive consensus.

$$
m_{\mathcal{M}^{f}(\Theta)}(A)=S_{1}(A) \triangleq \sum_{\substack{X_{1}, \ldots, X_{k} \in D^{\Theta} \\\left(X_{1} \cap \ldots, \ldots X_{k}\right)=A}} \prod_{i=1}^{k} m_{i}\left(X_{i}\right)
$$

* otherwise discounting techniques of the sources must be applied at first 


\section{On the associativity of DSm rules}

\section{The general/hybrid DSm rule of combination}

The general DSm rule of combination is commutative but is in general not associative.

$$
\underbrace{\left[m_{1} \oplus m_{2} \oplus m_{3}\right](.)}_{\text {Optimal Fusion }} \neq \underbrace{\left[\left(m_{1} \oplus m_{2}\right) \oplus m_{3}\right](.)}_{\text {Suboptimal fusion }} \neq \underbrace{\left[m_{1} \oplus\left(m_{2} \oplus m_{3}\right)\right](.)}_{\text {Suboptimal fusion }} \neq \underbrace{\left[m_{2} \oplus\left(m_{1} \oplus m_{3}\right)\right](.)}_{\text {Suboptimal fusion }}
$$

This is normal. To preserve optimality and coherence of the fusion result, all the sources have to be combined together at same fusion level (centralized fusion), not sequentially. Sequential/decentralized fusion is only suboptimal since part of information is lost during intermediate fusion steps.

Special case : The classic DSm rule of combination (for free DSm model)

Classic DSm rule (i.e. conjunctive consensus) over hyper-power set is commutative and associative. 


\section{DST and Dempster's rule of combination}

\section{Assumption: Shafier's model (close world assumption + refilinement)}

Basic belief assignment (bba):

$$
m(.): 2^{\Theta} \rightarrow[0,1]
$$

$$
m(\emptyset)=0 \quad \text { and } \quad \sum_{A \in 2^{\Theta}} m(A)=1
$$

Credibility: $\operatorname{Bel}(A)=\sum_{B \in 2^{\ominus}, B \subseteq A} m(B)$

Plausibility:

$$
\operatorname{Pl}(A)=\sum_{B \in 2^{\ominus}, B \cap A \neq \emptyset} m(B)
$$

Dempster's rule: Fusion of 2 independent equally reliable sources with bba $m_{1}$ and $m_{2}$
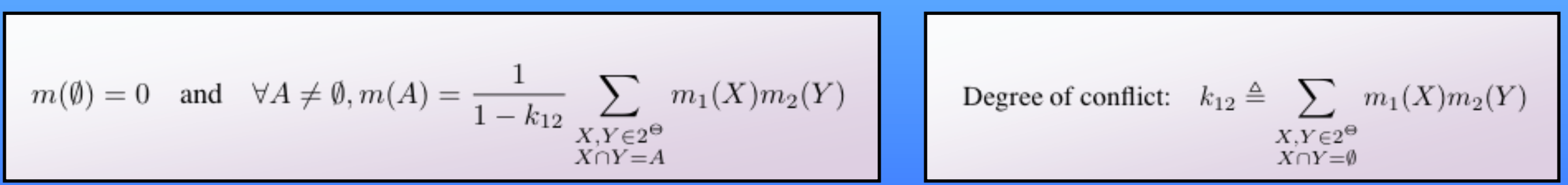

Dempster's rule generates counter-intuitive results when conflict becomes high and is even not defined $(0 / 0)$ when $k_{12}=1$ (sources in full contradiction). Problem arises not only from reliability of sources but is due also to the limited knowledge or experience of sources/experts to build their bba and their own interpretation of elements of the frame.

Classical issues:

1 - choose some heuristic thresholding techniques on $k_{12}$ to accept fusion result 2 - modify the discounting factors

3 - Adopt one of existing alternative rules proposed in literature (disjunctive, Yager, Dubois \& Prade, Smets open-world approach)

But how to prevent fusion troubles with the Dempster's rule beforehand ?

$\rightarrow$ Switch for a new competitive theory to eliminate inherent and potential troubles 


\section{Main existing alternatives to Dempster's rule}

Assumption: Shafer's model

Yager's rule:

[Yager 1983]

Disjunctive rule:

[Dubois \& Prade 1986]

Dubois \& Prade's (hybrid) rule:

[Dubois \& Prade 1988]

\section{Assumption: Open-world}

Smets' rule: [Smets 1994]

Smets' rule is the non-normalized version of the Dempster's rule.

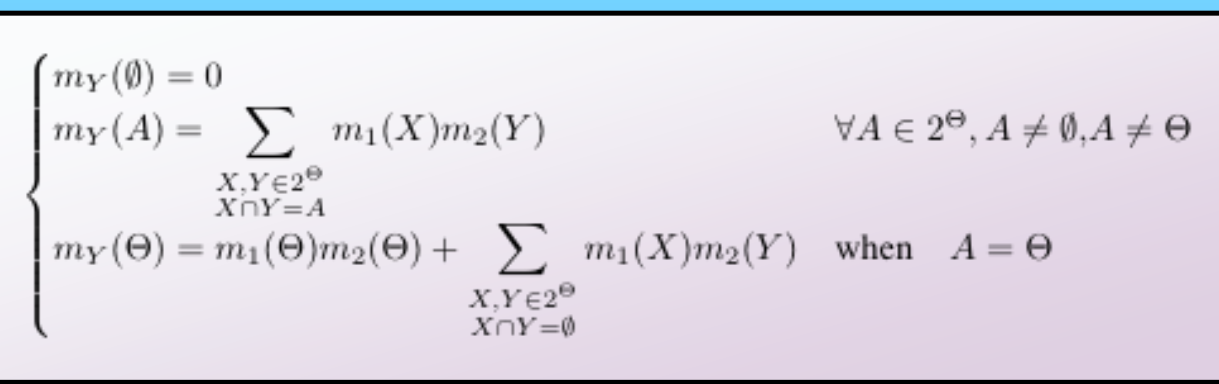

$$
m_{\cup}(\emptyset)=0 \quad \text { and } \forall A \neq \emptyset, m_{\cup}(A)=\sum_{\substack{X, Y \in 2 \\ X \cup Y=A}} m_{1}(X) m_{2}(Y)
$$

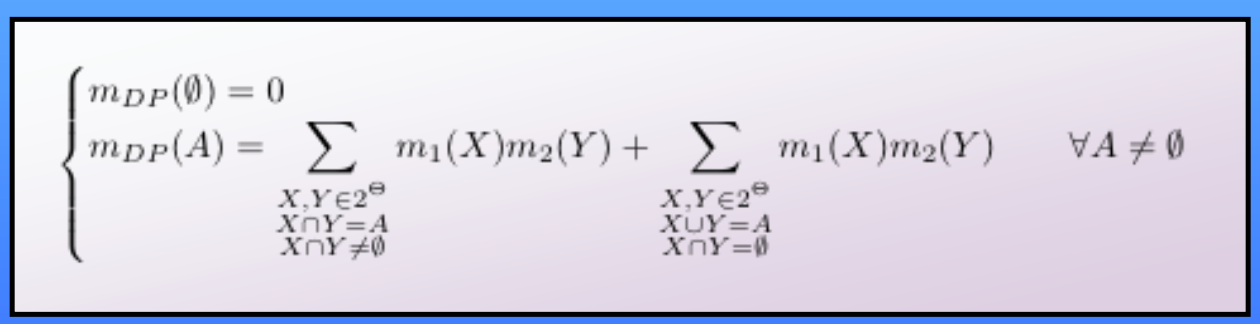

One allows positive bba on empty set

$$
\left\{\begin{array}{l}
m_{S}(\emptyset) \equiv k_{12}=\sum_{\substack{X, Y \in 2^{\ominus} \\
X \cap Y=\emptyset}} m_{1}(X) m_{2}(Y) \\
m_{S}(A)=\sum_{\substack{X, Y \in 2^{\ominus} \\
X \cap Y=A}} m_{1}(X) m_{2}(Y) \quad \forall A \neq \emptyset
\end{array}\right.
$$

Note : A general unified formulation exists for all rules involving conjunctive consensus (Dempster's, Smets', Yager's, Dubois \& Prade's, etc). [Lefevre et al. 2002] 


\section{Comments on refinement}

$$
\begin{array}{lll}
\Theta=\left\{\theta_{1}=\text { Small, } \theta_{2}=\text { Tall }\right\} . & \\
m_{1}\left(\theta_{1}\right)=0.4 & m_{1}\left(\theta_{2}\right)=0.5 & m_{1}\left(\theta_{1} \cup \theta_{2}\right)=0.1 \\
m_{2}\left(\theta_{1}\right)=0.6 & m_{2}\left(\theta_{2}\right)=0.2 & m_{2}\left(\theta_{1} \cup \theta_{2}\right)=0.2
\end{array}
$$

Case 1: Assumption = Shafer's model holds

Dempster's rule yields: $k_{12}=m_{1}\left(\theta_{1}\right) m_{2}\left(\theta_{2}\right)+m_{2}\left(\theta_{1}\right) m_{1}\left(\theta_{2}\right)=0.38$

$$
m(\emptyset)=0 \quad m\left(\theta_{1}\right)=\frac{0.38}{1-0.38}=0.613 \quad m\left(\theta_{2}\right)=\frac{0.22}{1-0.38}=0.355 \quad m\left(\theta_{1} \cup \theta_{2}\right)=\frac{0.02}{1-0.38}=0.032
$$

General/hybrid DSm rule yields: $\quad$ DSm rule is not equivalent to Dempster's rule

$$
m(\emptyset)=0 \quad m\left(\theta_{1}\right)=0.38 \quad m\left(\theta_{2}\right)=0.22 \quad m\left(\theta_{1} \cup \theta_{2}\right)=0.02+0.38=0.40
$$

For this simple 2D static fusion problem, the general DSm rule coincides with Yager's and Dubois \& Prade's rules of combination.

Question : What to do if one does not accept the previous Shafer's model (because of the continuity of elements of the frame and their relative interpretation) ? 


\section{Comments on refinement (cont'd)}

Case 2: Assumption = Shafer's model does not hold (one accepts the free DSm model)

Classical DSm rule yields directly:

$$
m(\emptyset)=0 \quad m\left(\theta_{1} \cap \theta_{2}\right)=0.38 \quad m\left(\theta_{1}\right)=0.38 \quad m\left(\theta_{2}\right)=0.22 \quad m\left(\theta_{1} \cup \theta_{2}\right)=0.02
$$

Starting with same bba as inputs and assume now that a refined frame exists in order to apply Dempster's rule, i.e.

$$
\begin{aligned}
& \theta_{1} \cap \theta_{2}=\theta_{2}^{\prime} \\
& \theta_{2}=\theta_{2}^{\prime} \cup \theta_{3}^{\prime} \\
& \theta_{1}=\theta_{1}^{\prime} \cup \theta_{2}^{\prime}
\end{aligned}
$$

$$
\begin{array}{lll}
\Theta_{\text {ref }} \triangleq\left\{\theta_{1}^{\prime}=\text { Small', } \theta_{2}^{\prime} \triangleq\right. \text { Medium, } & \left.\theta_{3}^{\prime}=\text { Tall' }\right\} \\
m_{1}^{\prime}\left(\theta_{1}^{\prime} \cup \theta_{2}^{\prime}\right)=0.4 & m_{1}^{\prime}\left(\theta_{2}^{\prime} \cup \theta_{3}^{\prime}\right)=0.5 & m_{1}^{\prime}\left(\theta_{1}^{\prime} \cup \theta_{2}^{\prime} \cup \theta_{3}^{\prime}\right)=0.1 \\
m_{2}^{\prime}\left(\theta_{1}^{\prime} \cup \theta_{2}^{\prime}\right)=0.6 & m_{2}^{\prime}\left(\theta_{2}^{\prime} \cup \theta_{3}^{\prime}\right)=0.2 & m_{2}^{\prime}\left(\theta_{1}^{\prime} \cup \theta_{2}^{\prime} \cup \theta_{3}^{\prime}\right)=0.2
\end{array}
$$

Dempster's rule on the refined frame yields:

(there is now no conflicting mass)

$\begin{aligned} m(\emptyset) & =0 \\ m\left(\theta_{2}^{\prime}\right) & =m_{1}^{\prime}\left(\theta_{1}^{\prime} \cup \theta_{2}^{\prime}\right) m_{2}^{\prime}\left(\theta_{2}^{\prime} \cup \theta_{3}^{\prime}\right)+m_{2}^{\prime}\left(\theta_{1}^{\prime} \cup \theta_{2}^{\prime}\right) m_{1}^{\prime}\left(\theta_{2}^{\prime} \cup \theta_{3}^{\prime}\right)=0.38 \\ m\left(\theta_{1}^{\prime} \cup \theta_{2}^{\prime}\right) & =m_{1}^{\prime}\left(\theta_{1}^{\prime} \cup \theta_{2}^{\prime}\right) m_{2}^{\prime}\left(\theta_{1}^{\prime} \cup \theta_{2}^{\prime}\right)+m_{1}^{\prime}\left(\theta_{1}^{\prime} \cup \theta_{2}^{\prime} \cup \theta_{3}^{\prime}\right) m_{2}^{\prime}\left(\theta_{1}^{\prime} \cup \theta_{2}^{\prime}\right)+m_{2}^{\prime}\left(\theta_{1}^{\prime} \cup \theta_{2}^{\prime} \cup \theta_{3}^{\prime}\right) m_{1}^{\prime}\left(\theta_{1}^{\prime} \cup \theta_{2}^{\prime}\right)=0.38 \\ m\left(\theta_{2}^{\prime} \cup \theta_{3}^{\prime}\right) & =m_{1}^{\prime}\left(\theta_{2}^{\prime} \cup \theta_{3}^{\prime}\right) m_{2}^{\prime}\left(\theta_{2}^{\prime} \cup \theta_{3}^{\prime}\right)+m_{1}^{\prime}\left(\theta_{1}^{\prime} \cup \theta_{2}^{\prime} \cup \theta_{3}^{\prime}\right) m_{2}^{\prime}\left(\theta_{2}^{\prime} \cup \theta_{3}^{\prime}\right)+m_{2}^{\prime}\left(\theta_{1}^{\prime} \cup \theta_{2}^{\prime} \cup \theta_{3}^{\prime}\right) m_{1}^{\prime}\left(\theta_{2}^{\prime} \cup \theta_{3}^{\prime}\right)=0.22 \\ m\left(\theta_{1}^{\prime} \cup \theta_{2}^{\prime} \cup \theta_{3}^{\prime}\right) & =m_{1}^{\prime}\left(\theta_{1}^{\prime} \cup \theta_{2}^{\prime} \cup \theta_{3}^{\prime}\right) m_{2}^{\prime}\left(\theta_{1}^{\prime} \cup \theta_{2}^{\prime} \cup \theta_{3}^{\prime}\right)=0.02\end{aligned}$

Thus the Dempster's rule reduces to Classical DSm rule but with the necessity of existence and justification of possible refinement. It introduces useless complexity w.r.t the DSm formalism. 


\section{Static and dynamic Fusion}

Based on the same general formalism DSmT is well adapted to deal with:

1- static fusion applications (i.e. the frame and model do not change with time).

2- dynamic fusion applications (i.e. the frame and/or model vary with time) with the use of the proper characteristic non-emptiness function.

3 - Fusion over different frames by min. specificity extension [Smets 1993] Simple example of dynamic fusion (testimony problem)

$$
\Theta\left(t_{l}\right) \triangleq\left\{\theta_{1} \equiv \text { young, } \theta_{2} \equiv \text { old, } \theta_{3} \equiv \text { white hairs }\right\}
$$
Witnesses reports

$\begin{array}{ll}m_{1}\left(\theta_{1}\right)=0.5 & m_{1}\left(\theta_{3}\right)=0.5 \\ m_{2}\left(\theta_{2}\right)=0.5 & m_{2}\left(\theta_{3}\right)=0.5\end{array}$

Applying the general DSm rule (based on free DSm model), one gets:

$$
m_{\mathcal{M}^{f}\left(\Theta\left(t_{l}\right)\right)}\left(\theta_{1} \cap \theta_{2}\right)=0.25 \quad m_{\mathcal{M}^{f}\left(\Theta\left(t_{l}\right)\right)}\left(\theta_{1} \cap \theta_{3}\right)=0.25 \quad m_{\mathcal{M}^{f}\left(\Theta\left(t_{l}\right)\right)}\left(\theta_{2} \cap \theta_{3}\right)=0.25 \quad m_{\mathcal{M}^{f}\left(\Theta\left(t_{l}\right)\right)}\left(\theta_{3}\right)=0.25
$$

If one learns that young people don't have white hairs, one introduces this integrity constraint in the model, by setting:

$$
\Phi\left(\theta_{1} \cap \theta_{3}\right) \stackrel{\mathcal{M}}{=} 0
$$

Applying the general DSm rule, one gets now:

$$
m_{\mathcal{M}\left(\Theta\left(t_{l+1}\right)\right)}\left(\theta_{3}\right)=0.25 \quad m_{\mathcal{M}\left(\Theta\left(t_{i+1}\right)\right)}\left(\theta_{1} \cap \theta_{2}\right)=0.25 \quad m_{\mathcal{M}\left(\Theta\left(t_{l+1}\right)\right)}\left(\theta_{2} \cap \theta_{3}\right)=0.25 \quad m_{\mathcal{M}\left(\Theta\left(t_{i+1}\right)\right)}\left(\theta_{1} \cup \theta_{3}\right)=0.25
$$




\section{DST vs. DSmT - Examples}

\begin{tabular}{|c|c|c|c|}
\hline Frame & $\Theta=\left\{\theta_{1}, \theta_{2}, \theta_{3}\right\}$ & Inputs & $\begin{array}{lll}m_{1}\left(\theta_{1}\right)=1-\epsilon_{1} & m_{1}\left(\theta_{2}\right)=0 & m_{1}\left(\theta_{3}\right)=\epsilon_{1} \\
m_{2}\left(\theta_{1}\right)=0 & m_{2}\left(\theta_{2}\right)=1-\epsilon_{2} & m_{2}\left(\theta_{3}\right)=\epsilon_{2}\end{array}$ \\
\hline
\end{tabular}

\section{Dempster's rule}

(Shafer's model)

$$
m\left(\theta_{3}\right)=\frac{e_{1} e_{2}}{\left(1-e_{1}\right) \cdot 0+0 \cdot\left(1-e_{2}\right)+e_{1} e_{2}}=1
$$

When $0<e_{1}<1$ and $0<e_{2}<1$, Dempster's rule provides in this specific case same result whatever the values of $e_{1}$ and $e_{2}$ are !!! Dempster's rule is mathematically not defined when $e_{1}=e_{2}=0$.

It provides only a coherent and trivial solution when $e_{1}=e_{2}=1$.

\section{Classic DSm rule (free DSm model)}

$$
m\left(\theta_{3}\right)=e_{1} e_{2} \quad m\left(\theta_{1} \cap \theta_{2}\right)=\left(1-e_{1}\right)\left(1-e_{2}\right) \quad m\left(\theta_{1} \cap \theta_{3}\right)=\left(1-e_{1}\right) e_{2} \quad m\left(\theta_{2} \cap \theta_{3}\right)=\left(1-e_{2}\right) e_{1}
$$

General DSm rule (one adopts the Shafer's model)

$$
m\left(\theta_{3}\right)=e_{1} e_{2} \quad m\left(\theta_{1} \cup \theta_{2}\right)=\left(1-e_{1}\right)\left(1-e_{2}\right) \quad m\left(\theta_{1} \cup \theta_{3}\right)=\left(1-e_{1}\right) e_{2} \quad m\left(\theta_{2} \cup \theta_{3}\right)=\left(1-e_{2}\right) e_{1}
$$

DSmT provides coherent result depending on $e_{1}$ and $e_{2}$. $e_{1}$ and $e_{2}$ can take any values in $[0,1]$ 


\section{DST vs. DSmT - Examples (cont'd)}

Frame

$$
\Theta=\left\{\theta_{1}, \theta_{2}, \theta_{3}, \theta_{4}\right\} \quad \text { Inputs }
$$

$$
\begin{array}{ll}
m_{1}\left(\theta_{1}\right)=0.99 & m_{1}\left(\theta_{3} \cup \theta_{4}\right)=0.01 \\
m_{2}\left(\theta_{2}\right)=0.98 & m_{2}\left(\theta_{3} \cup \theta_{4}\right)=0.02
\end{array}
$$

\section{Dempster's rule}

(Shafer's model)

$$
m\left(\theta_{3} \cup \theta_{4}\right)=\frac{(0.01 \cdot 0.02)}{(0+0+0+0+0.01 \cdot 0.02)}=1
$$

\section{Classic DSm rule (free DSm model)}

$$
m\left(\theta_{1} \cap \theta_{2}\right)=0.9702 \quad m\left(\theta_{1} \cap\left(\theta_{3} \cup \theta_{4}\right)\right)=0.0198 \quad m\left(\theta_{2} \cap\left(\theta_{3} \cup \theta_{4}\right)\right)=0.0098 \quad m\left(\theta_{3} \cup \theta_{4}\right)=0.0002
$$

General DSm rule (one adopts the Shafer's model)

$$
m\left(\theta_{1} \cup \theta_{2}\right)=0.9702 \quad m\left(\theta_{1} \cup \theta_{3} \cup \theta_{4}\right)=0.0198 \quad m\left(\theta_{2} \cup \theta_{3} \cup \theta_{4}\right)=0.0098 \quad m\left(\theta_{3} \cup \theta_{4}\right)=0.0002
$$

DSmT still provides a coherent result

There exist several infinite classes of fusion problems where the Dempster's rule fails to provide coherent results (or at least counter-intuitive results) [Dezert/Smarandache/Khosnevisan 2004] 


\section{Comparisons of fusion rules}

\begin{tabular}{|c|c|c|}
\hline Frame & $\Theta=\left\{\theta_{1}, \theta_{2}, \theta_{3}\right\}$ & $\begin{array}{llll}m_{1}\left(\theta_{1}\right)=0.1 & m_{1}\left(\theta_{2}\right)=0.4 & m_{1}\left(\theta_{3}\right)=0.2 & m_{1}\left(\theta_{1} \cup \theta_{2}\right)=0.3 \\
m_{2}\left(\theta_{1}\right)=0.5 & m_{2}\left(\theta_{2}\right)=0.1 & m_{2}\left(\theta_{3}\right)=0.3 & m_{2}\left(\theta_{1} \cup \theta_{2}\right)=0.1\end{array}$ \\
\hline
\end{tabular}

\section{Consider the Dynamic fusion problem with non-existential constraint}

New info.: The third suspect provides a strong alibi

$$
\theta_{3} \stackrel{\mathcal{M}}{=} \emptyset \text { (extra integrity constraint) }
$$

\section{Classic DSm rule (free DSm model)}

$\begin{array}{llll}m\left(\theta_{1}\right)=0.21 & m\left(\theta_{2}\right)=0.11 & m\left(\theta_{3}\right)=0.06 & m\left(\theta_{1} \cup \theta_{2}\right)=0.03 \\ m\left(\theta_{1} \cap \theta_{2}\right)=0.21 & m\left(\theta_{1} \cap \theta_{3}\right)=0.13 & m\left(\theta_{2} \cap \theta_{3}\right)=0.14 & m\left(\theta_{3} \cap\left(\theta_{1} \cup \theta_{2}\right)\right)=0.11\end{array}$

General DSm rule (hybrid DSm model = Shafer's model + extra constraint)

The conflicting mass to transfer is then

$$
k_{12}=0.06+0.21+0.13+0.14+0.11=0.65
$$

Fusion result

$$
m(\emptyset)=0 \quad m\left(\theta_{1}\right)=0.34 \quad m\left(\theta_{2}\right)=0.25 \quad m\left(\theta_{1} \cup \theta_{2}\right)=0.41
$$

Smets' rule

$$
m_{S}(\emptyset)=0.65 \quad m_{S}\left(\theta_{1}\right)=0.21 \quad m_{S}\left(\theta_{2}\right)=0.11 \quad m_{S}\left(\theta_{1} \cup \theta_{2}\right)=0.03
$$




\section{Comparisons of fusion rules (cont'd)}

Yager's rule

$$
m_{Y}(\emptyset)=0 \quad m_{Y}\left(\theta_{1}\right)=0.21 \quad m_{Y}\left(\theta_{2}\right)=0.11 \quad m_{Y}\left(\theta_{1} \cup \theta_{2}\right)=0.03+k_{12}=0.03+0.65=0.68
$$

\section{Dempster's rule}

$$
m_{D S}(\emptyset)=0 \quad m_{D S}\left(\theta_{1}\right)=\frac{0.21}{1-0.65}=0.60 \quad m_{D S}\left(\theta_{2}\right)=\frac{0.11}{1-0.65} \approx 0.314 \quad m_{D S}\left(\theta_{1} \cup \theta_{2}\right)=\frac{0.03}{1-0.65} \approx 0.086
$$

\section{Dubois \& Prade's rule}

\section{If one adds up masses one gets $0.94<1$}

$$
\begin{aligned}
& m_{D P}(\emptyset)=0 \\
& m_{D P}\left(\theta_{1}\right)=\left[m_{1}\left(\theta_{1}\right) m_{2}\left(\theta_{1}\right)+m_{1}\left(\theta_{1}\right) m_{2}\left(\theta_{1} \cup \theta_{2}\right)+m_{2}\left(\theta_{1}\right) m_{1}\left(\theta_{1} \cup \theta_{2}\right)\right]+\left[m_{1}\left(\theta_{1}\right) m_{2}\left(\theta_{3}\right)+m_{2}\left(\theta_{1}\right) m_{1}\left(\theta_{3}\right)\right]=0.34 \\
& m_{D P}\left(\theta_{2}\right)=\left[m_{1}\left(\theta_{2}\right) m_{2}\left(\theta_{2}\right)+m_{1}\left(\theta_{2}\right) m_{2}\left(\theta_{1} \cup \theta_{2}\right)+m_{2}\left(\theta_{2}\right) m_{1}\left(\theta_{1} \cup \theta_{2}\right)\right]+\left[m_{1}\left(\theta_{2}\right) m_{2}\left(\theta_{3}\right)+m_{2}\left(\theta_{2}\right) m_{1}\left(\theta_{3}\right)\right]=0.25 \\
& m_{D P}\left(\theta_{1} \cup \theta_{2}\right)=\left[m_{1}\left(\theta_{1} \cup \theta_{2}\right) m_{2}\left(\theta_{1} \cup \theta_{2}\right)\right]+\left[m_{1}\left(\theta_{1} \cup \theta_{2}\right) m_{2}\left(\theta_{3}\right)+m_{2}\left(\theta_{1} \cup \theta_{2}\right) m_{1}\left(\theta_{3}\right)\right]+\left[m_{1}\left(\theta_{1}\right) m_{2}\left(\theta_{2}\right)+m_{2}\left(\theta_{1}\right) m_{1}\left(\theta_{2}\right)\right]=0.35
\end{aligned}
$$

Dubois \& Prade's rule doesn't work for dynamic fusion problems when a singleton or an union of singletons becomes empty. This problem is fixed by the sum $\mathrm{S}_{2}$ in the general DSm rule of combination.

When there is no non-existential constraint, the general DSm rule is consistent with Dubois \& Prade's rule. 


\section{Extension of DSm rule for imprecise beliefs}

Operations on sets

\author{
Addition \\ Subtraction \\ Multiplication
}

$$
\begin{aligned}
& S_{1} \boxplus S_{2}=S_{2} \boxplus S_{1} \triangleq\left\{x \mid x=s_{1}+s_{2}, s_{1} \in S_{1}, s_{2} \in S_{2}\right\} \\
& S_{1} \boxminus S_{2} \triangleq\left\{x \mid x=s_{1}-s_{2}, s_{1} \in S_{1}, s_{2} \in S_{2}\right\} \\
& S_{1} \boxminus S_{2} \triangleq\left\{x \mid x=s_{1} \cdot s_{2}, s_{1} \in S_{1}, s_{2} \in S_{2}\right\}
\end{aligned}
$$

Inputs Imprecise admissible generalized basic belief assignments $\mathrm{m}^{\mathrm{I}}(\mathrm{.})$ of the form

$m^{I}(A)=\left[a_{1}, b_{1}\right] \cup \ldots \cup\left[a_{m}, b_{m}\right] \cup\left(c_{1}, d_{1}\right) \cup \ldots \cup\left(c_{n}, d_{n}\right) \cup\left(e_{1}, f_{1}\right] \cup \ldots \cup\left(e_{p}, f_{p}\right] \cup\left[g_{1}, h_{1}\right) \cup \ldots \cup\left[g_{q}, h_{q}\right) \cup\left\{A_{1}, \ldots, A_{r}\right\}$ where all the bounds or elements involved into $m^{I}(A)$ belong to $[0,1]$

Extented general DSm rule

\section{A simple 2D example}

\begin{tabular}{|c|c|c|}
\hline$A \in D^{\Theta}$ & $m_{1}^{I}(A)$ & $m_{2}^{I}(A)$ \\
\hline$\theta_{1}$ & {$[0.1,0.2] \cup\{0.3\}$} & {$[0.4,0.5]$} \\
$\theta_{2}$ & $(0.4,0.6) \cup[0.7,0.8]$ & {$[0,0.4] \cup\{0.5,0.6\}$} \\
\hline
\end{tabular}

Inputs

$$
m_{\mathcal{M}(\Theta)}^{I}(A) \triangleq \phi(A) \square\left[S_{1}^{I}(A) \boxplus S_{2}^{I}(A) \boxplus S_{3}^{I}(A)\right]
$$

Fusion on free DSm model

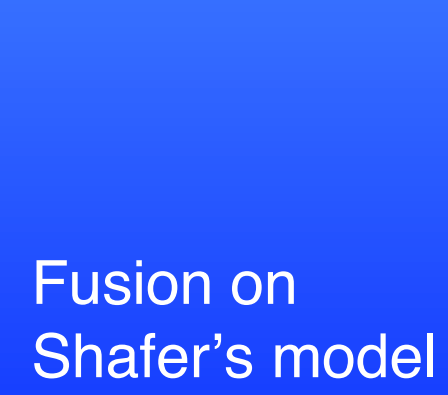

\begin{tabular}{|c|c|}
\hline$A \in D^{\Theta}$ & $m_{\mathcal{M}}^{I}(A)=\left[m_{1}^{I} \oplus m_{2}^{I}\right](A)$ \\
\hline$\theta_{1}$ & {$[0.04,0.10] \cup[0.12,0.15]$} \\
$\theta_{2}$ & {$[0,0.40] \cup[0.42,0.48]$} \\
$\theta_{1} \cap \theta_{2} \stackrel{\mathcal{M}}{\equiv} \emptyset$ & 0 \\
$\theta_{1} \cup \theta_{2}$ & $(0.16,0.58]$ \\
\hline
\end{tabular}




\section{Summary of the DSm Fusion Principle}

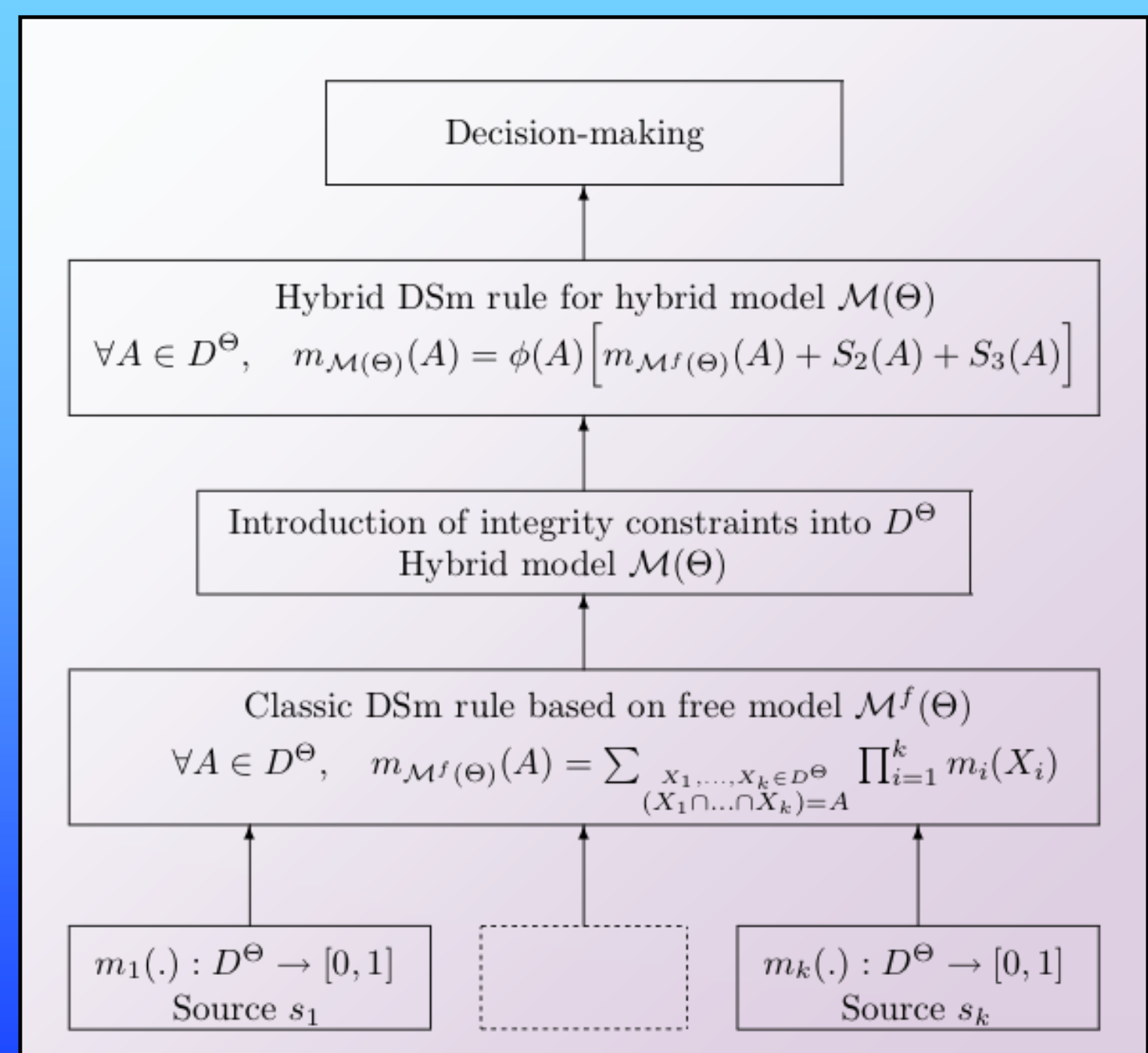




\section{Practical limitations of DSmT}

Complexity of the general DSm rule w.r.t. all previous existing rules of combination. This is the price to pay ....

DSmT is useful for problems of small dimension but becomes intractable for problems of large dimension in the worst case (i.e. when the core of bba coincides with hyper-power set) since the cardinality of hyper-power set increases rapidly (the cardinality however remains much smaller than the cardinality of the power set of the refined frame, when refinement is possible)

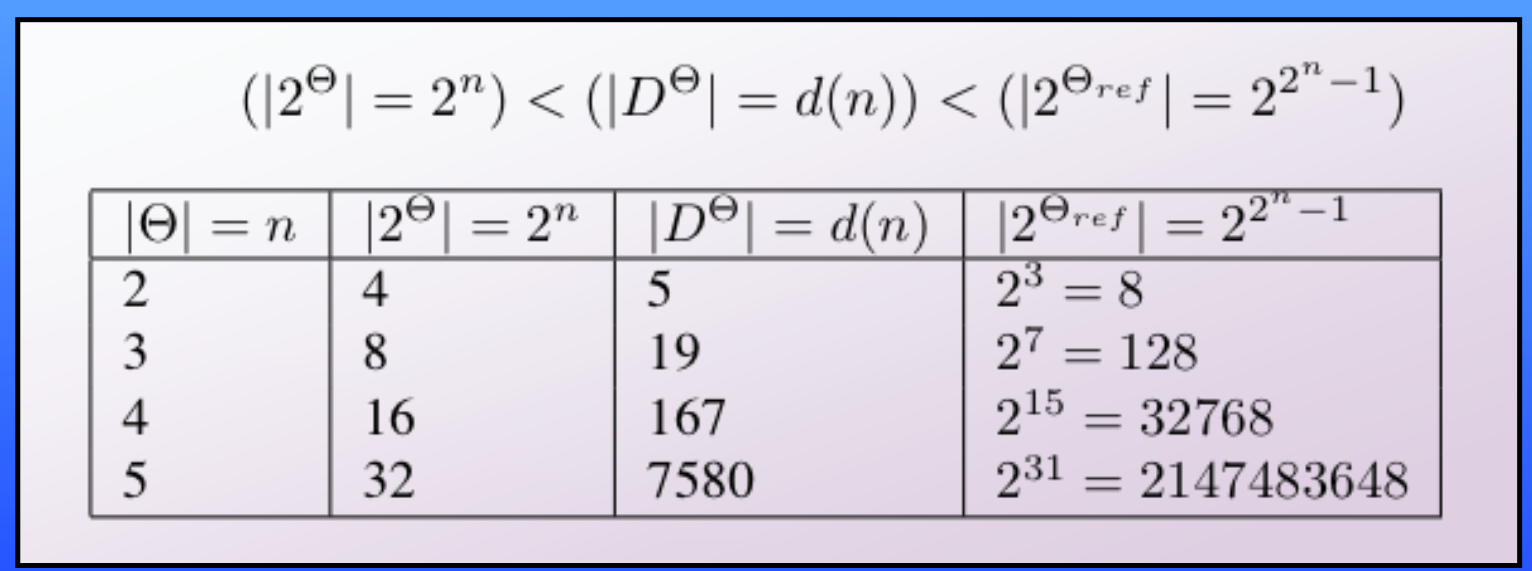

There is no consensus for decision-making in DSmT framework (same problem exists within DST framework). Many criteria can actually be adopted (GPT is only an issue). 


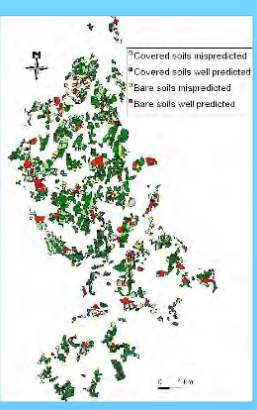

\section{Recent applications of DSmT}

\section{Land cover change prediction for pollution prevention [Corgne et al. 2004]}

SPOT + IRS-LISS III images + GIS + agricultural experts

\section{Power and resource aware distributed smart fusion [Kadambe 2004]}

Optimization of disparate DSN architecture to minimize power consumption and optimize multitarget detection and classification (typical application of dynamic fusion).

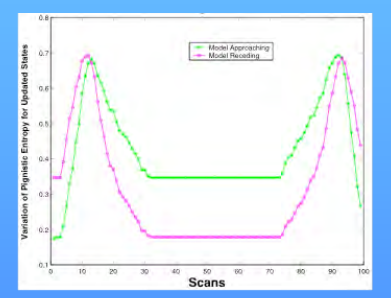

Estimation of target behavior tendencies [Tchamova et. al 2003]

Sonar amplitude measurements + fuzzification interface + DSmT

Generalized data association for MTT in clutter [Tchamova et. al 2004]

Multitarget tracking with kinematics and attribute measurements
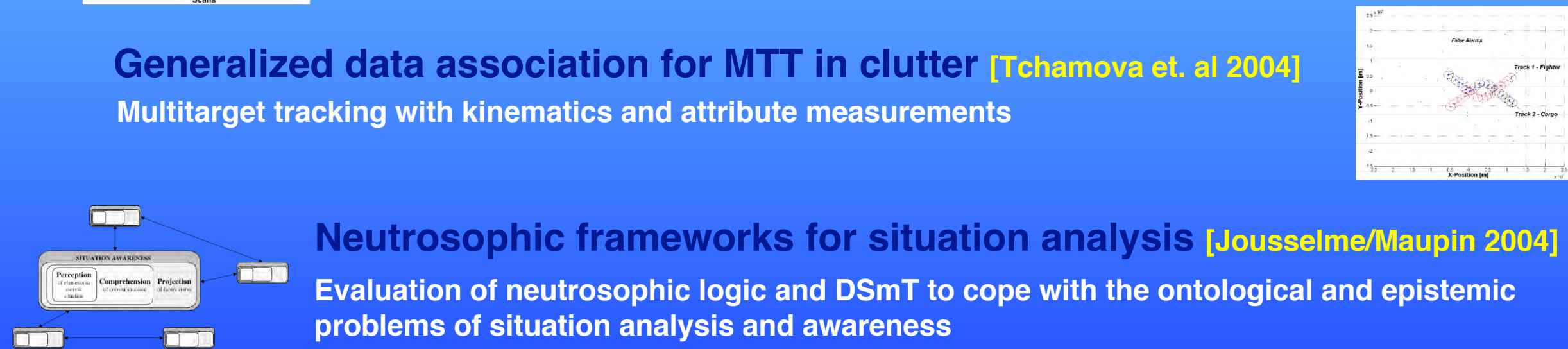

Neutrosophic frameworks for situation analysis [Jousselme/Maupin 2004]

Evaluation of neutrosophic logic and DSmT to cope with the ontological and epistemic problems of situation analysis and awareness

Solution to the Tweety Penguin Triangle Problem [Dezert/Smarandache 2004] Analysis and comparison of Bayesian, Shaferian reasonings w.r.t. DSmT within weighted contradicting rules-based systems.

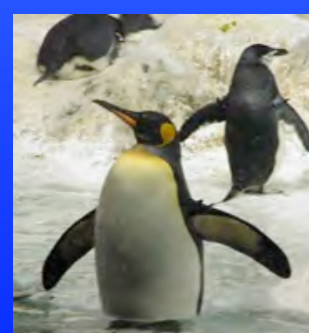




\section{Conclusions}

- DSmT proposes new mathematical foundations for information fusion and is a new competitive alternative to DST with following specificities:

- It can work beyond the limits of the applicability of the DST

Q It takes into account the intrinsic nature/granularity of information

- It works on any models (including Shafer's model)

- It provides a new fusion rule able to deal with static or dynamic fusion problems expressed in terms of belief functions and based on the same general formalism

- It allows to combine uncertain, highly conflicting and imprecise information provided by independent sources of evidence

$@$ Reliability (when known) of sources can be easily taken into account

- It is a natural extension of previous works done by Yager, Dubois \& Prade , Smets and others to circumvent DST limitations.

- Potentially, DSmT can be applied in mostfusion applications where DST has already been used so far, but it can also cover a wider class of applications because of its new appealing specificities.

Reference: "Advances and Applications of DSmT for Information Fusion (Collected works)"', American Research Press, Rehoboth, 418 p., 2004. 